\title{
La wilāyat al-faqih después de la Revolución. El vali-ye faqih en la Constitución y el nezām de la República islámica de Irán
}

\section{The Wilāyat al-Faqih After the Revolution. The Vali-ye Faqih in the Constitution and Nezām of the Islamic Republic of Iran}

\author{
Raffaele Mauriello ${ }^{1}$ \\ Universidad Allameh Tabataba’i, Teherán (Irán)
}

Recibido: 23-01-19

Aprobado: 02-03-19

\section{Resumen}

El presente ensayo trata la doctrina teológico-jurídica de la wilāyat al-faqih (en árabe, velāyat-e faqih en persa), expresión que puede traducirse con 'tutela del jurisperito islámico’ -y, en la rendición de la doctrina por parte del gran ayatola Jomeiní, hasta con 'gobierno del jurisperito islámico'-y su desarrollo e institucionalización en la República islámica de Irán (R.I. de Irán) después de la Revolución de 1979. En este marco, el ensayo analiza la función del vali-ye faqih, el jurisperito islámico encargado del gobierno de la R.I. de Irán, en la Constitución y el ordenamiento (nezām) del país. El término vali-ye faiqh, por su reiterado uso y prestigio, tiende a alternarse con sinónimos como rahbar, rahbar-e enqelāb, maqām-e rahbari o maqām-e mo'azzam-e rahbari.

Palabras-clave: Wilāyat al-faqih, nezām, Jomeiní, Jamenei, República Islámica de Irán, Islamología, Constitucionalismo.

\footnotetext{
${ }^{1}$ (raffaele.mauriello@me.com) Profesor ayudante doctor en la Facultad de Literatura Persa y Lenguas Extranjeras de la Universidad Allameh Tabataba'i y Profesor invitado en el Master en Geopolítica y Seguridad Global de la Universidad La Sapienza de Roma. Es Doctor en Civilización Islámica: Historia y Filología de la Universidad La Sapienza de Roma y tiene el título de Postdoctorado en Estudios sobre el Mundo de la Universidad de Teherán. Es cofundador del International Relations and Islamic Studies Research Cohort (Co-IRIS), codirector del International Journal of Islam in Asia (IJIA) publicado por Brill Publishers, corresponsable de la colección «Islam and International Relations» publicada por Gerlach Press y de la colección «Islam and Global Studies» publicada por Palgrave-Macmillan. En el año 2013 fue galardonado con el The World Prize for the Book of the Year of the Islamic Republic of Iran en el campo de la islamología por su monografía Descendants of the Familiy of the Prophet in Contemporary History: A Case Study, the Š $\check{\imath}_{i}$ Religious Establishment of al-Nağaf (Iraq).
} 


\begin{abstract}
This essay deals with the theological-juridical doctrine of the Arabic wilàyat al-faqih (velāyat-e faqih in Persian), an expression that can be translated as 'tutelage of the Islamic jurisprudent' - and, in the forwarding of doctrine by the Great Ayatollah Khomeini, even with 'government of the Islamic jurisprudent' - and its development and institutionalization in the Islamic Republic of Iran (IR of Iran) after the 1979 Revolution. In this framework, the essay analyzes the function of the vali-e faqih (the Islamic jurist in charge of the government of the RI of Iran), vis-a-vis the Constitution and the order (nezām) of the country. The term vali-ye faiqh, due to its repeated use and prestige, tends to alternate with synonyms like rahbar, rahbar-e enqelāb, maqām-e rahbari or maqām-e mo'azzam-e rahbari.
\end{abstract}

Key-words: Wilāyat al-faqih, nezām, Khomeini, Khamenei, Islamic Republic of Iran, Islamology, Constitutionalism.

\title{
Introducción
}

Una de las dos características más importantes del chiismo² imamí-la forma de chiismo mayoritaria en Irán- es la doctrina del imamato. Según esta doctrina, el profeta Mahoma designó explícitamente a su primo y yerno Ali como sucesor en la guía (política y religiosa) de la comunidad de los creyentes. A su vez, Ali designó a su hijo al-Hasan hasta llegar, de la misma forma y a través de una designación de padre en hijo, al último imam, el XII, al-Mahdi. Los imames no llevan nuevas revelaciones de naturaleza profética y desenvuelven más bien un papel fundamental como intérpretes de la profecía. Sin embargo, su capacidad de intérpretes tiene una peculiaridad significativa: en su interpretación ellos son infalibles ${ }^{3}$; y esta es la segunda característica fundamental del chiismo imamí. Por razones políticas, en el siglo IX el Duodécimo imam desaparece de la mirada de los hombres y desde entonces los chiíes se han puesto el problema de establecer sobre quién recae la responsabilidad del imam en su ausencia.

En el curso de los siglos y con diferentes matices, los jurisperitos islámicos ('ulamā' en árabe, 'olemā o ruhāni en persa) se han arrogado una parte de las prerrogativas de los imames como sus representantes (na i ib al-imām), en particular a través del concepto jurídico de la wilāyat al-faqih, la 'tutela del jurisperito islámico'. Durante muchos siglos, esta expresión ha sido en la mayoría de los casos -aunque no siempre-interpretada en el sentido de lo que

\footnotetext{
${ }^{2}$ Para simplificar la lectura de texto, en este ensayo se utilizará el término genérico 'chiismo', pero el ensayo trata del chiismo imamí y no de otras corrientes como el ibadí, ismailí, etc.

3 Alessandro Bausani, L’Islam, Milano, Garzanti, 1999, pp. 97-98.
} 
se llama al-wilāya al-jāssa, o sea la tutela de los huérfanos, los incapacitados, los enfermos y, en general, de la comunidad de los creyentes chíes contra los atropellos de la (ilegítima) autoridad en el poder ${ }^{4}$.

En los últimos siglos, y en particular en el siglo pasado, en el chiismo la organización interna de los 'ulam $\bar{a}$ ' se ha ido estructurando en una hierocracia ${ }^{5}$ a través del desarrollo de la marŷa 'iya (en árabe, marŷa 'iyat en persa). Esta es la guía religiosa (y en algunos, muy limitados, casos política) de la comunidad de los creyentes (chiíes), a base de una doctrina jurídica que prevé que todos los musulmanes (chiíes) estén obligados a seguir los dictámenes islámicos así como interpretados por parte de un marŷa 'al-taqlid ('fuente de emulación' o 'fuente de imitación'; para referirse a los marāŷa' al-taqlid [plural de marŷa ' al-taqlid] también se utiliza la locución ayatola al- 'uzmá, o 'gran ayatola'). Este último es un jurisperito islámico que haya efectuado sus estudios teológicos y jurídicos en la hawza 'ilmiyya (en árabe, house-ye 'elmiye en persa), o sea una de las "academias" tradicionales de estudio del islam chií situadas en las ciudades de al-Nayaf, Qom y Mashhad; y en algunos momentos de la historia del chiismo también en otras ciudades como Kerbala e Isfahán. Al final de estos estudios, al hombre, o mujer, que haya conseguido superar el tercer y último nivel se le atribuye el calificativo de muŷtahid/a o faqih/a (en algunos casos, también se utiliza el termino ayatola; sin embargo, este último es más ambiguo y controvertido, porque utilizado hoy en día también con valor político, por le menos en la R.I. de Irán) y se le otorga el permiso de efectuar el iŷtihād (o sea de interpretar los dictámenes de la tradición jurídica islámica ${ }^{6}$ ). El futuro marŷa al-taqlid debe, por una parte, haber conseguido este permiso y, por otra parte, y después de numerosos años, cuando no décadas, demostrar su efectiva capacidad de efectuar el î́tihād y ser reconocido como primus inter pares por parte de los otros marāŷy'al-taqlid. Finalmente, el que mira a esta posición, debe conseguir ser efectivamente seguido por un significativo numero de creyentes que pagan a

\footnotetext{
${ }^{4}$ Véase Abdulaziz Abdulhussein Sachedina, The Just Rules in Shi ite Islam: The Comprehensive Authority of the Jurut in Imamite Jurisprudence, New York, Oxford University Press, 1988. Según la teoría política clásica del chiismo, a parte por el imamato, las otras formas de autoridad política son ilegítimas.

${ }^{5}$ En este ensayo se ha optado por utilizar el término 'hierocracia' para referirse a la red y al sistema de oficios y "títulos" del establishment religioso chí, en lugar de 'clero' o 'Iglesia', en cuanto estos últimos, aun siendo útiles en un marco de divulgación científica, no son del todo adecuados para la descripción de este sistema en un ensayo académico. Este enfoque refleja en parte el de Said Amir Arjomand, The Turban for the Crown: The Islamic Revolution in Iran, New York, Oxford University Press, 1988, p. 7. Sin embargo, en sus trabajos más recientes Arjomand utiliza más bien el termino 'clero'. Véase por ejemplo Said Amir Arjomand, After Khomeini: Iran Under His Successors, New York, Oxford University Press, 2009.

${ }^{6}$ La posibilidad y necesidad de (re-)interpretación de la tradición jurídica islámica es una característica distintiva del islam chií y ha sido hasta incluida en la Constitución de la R.I. de Irán, en el art. 2, donde se afirma, entre otras cosas, que «La R.I. de Irán es un ordenamiento/Estado (nezām) basado en la fe: ... en el perpetuo iŷtihād de la escrituras por los foqahā (plural de faqih) dotados de todos los requisitos necesarios ( $\hat{y} \bar{a} m e$ 'ol-šarāyet)».
} 
él, o más bien a sus representantes, el sahm al-imam o jums, el impuesto islámico correspondiente al quinto de los ingresos ${ }^{7}$. Solo al final de este largo y difícil proceso es posible reclamar el "titulo" de marŷa al-taqlid.

Volviendo a la wilāyat al-faqih, la idea del gran ayatola Ruhollah Jomeiní es de extender a sus limites -y quizás un poquito más allá ${ }^{8}-$ la tradicional tutela del jurisperito islámico $\mathrm{y}$, sobretodo a través de su más radical elaboración doctrinal, la velāyat-e motlaqe-ye faqih ('tutela absoluta del jurisperito islámico'), de volver esta tutela en autoridad eminentemente política y de una "cualidad" no muy diferente de la del primer imam, Ali9. Según la doctrina política de la wilāyat al-faqih elaborada por Jomeiní, durante la ausencia del Duodécimo imam las leyes del islam no deben quedarse inaplicadas y la guía de la comunidad, el gobierno, corresponden a un jurisperito islámico cualificado -en un principio un marŷa 'al-taqlid, en una segunda reelaboración un faqih/muŷtahid dotado de algunas características especificas- que conoce la revelación divina y la voluntad de Dios ${ }^{10}$, y que tiene la responsabilidad de guiar a las masas hacia la realización del verdadero islam. A través de la reelaboración de la doctrina de la wilāyat al-faqih por parte de Jomeiní, desde ser limitado a algunos casos específicos de incapacitados, la función de tutela del faqih/muŷtahid se amplia hasta incluir todos los creyentes y, además, sale de la esfera estrictamente jurídica y se expande a las esferas políticas, sociales, culturales, económicas, etc.

\section{La wilāyat al-faqih en la Constitución de la República islámica de Irán}

Ha llegado ahora el momento de ver como esta doctrina, que está a la base del nezām ${ }^{11}$ de la República islámica de Irán (R.I. de Irán), ha tomado una

\footnotetext{
${ }^{7}$ Véase Linda S. Walbridge (ed.), The Most Learned of the Shi 'a: The Institution of the Marja ' al-Taqlid, New York, Oxford University Press, 2001.

8 Véase Hamid Mavani, "Analysis of Khomeini's Proofs for al-Wilaya al-Mutalqa [sic] (Comprehensive Authority) of the Jurist" [en Linda S. Walbridge, ed.: The Most Learned of the Shi 'a: The Institution of the Marja ' al-Taqlid, New York, Oxford University Press, 2001].

${ }^{9}$ Como descrito muy bien por Abdulaziz Abdulhussein Sachedina, The Just Rules in Shi ite Islam: The Comprehensive Authority of the Jurut in Imamite Jurisprudence, New York, Oxford University Press, 1988, a parte por el primer imam, Ali, los otros nunca gozaron de poder político.

10 Esta segunda también a través de su conocimiento trascendental. Al respecto, diversos estudiosos han puesto el acento en la visión gnóstica y en la influencia del pensamiento metafísico de la tradición chií representada en particular por Molla Sadra (¿1571?-1640) en el pensamiento, también jurídico y político, del gran ayatola Jomeiní. Véase por ejemplo Yahya Christian Bonaud, Uno gnóstico sconosciuto del XX secolo. Formazione e opere dell'Imam Khomeinî, a cura di Eugenio Tabano, Trieste, il Cerchio, 2010 [trad. de L'Imam Khomeyni, un gnostique méconnu du XXe siècle. Métaphysique et théologie dans les œuvres philosophiques et spirituelles de l'Imam Khomeyni, Beirut, Ed. Al-Bouraq, 1997].

${ }^{11}$ El termino nezām resulta fundamental en la descripción y análisis tanto de la institucionalización de la wilāyat al-faqih el la R.I. de Irán como en cualquier otro aspecto de la política del país. Sin embargo, nadie se ha ocupado de estudiar el valor de este termino. En la gran mayoría de los casos,
} 
forma concreta y jurídica en la Constitución del país ${ }^{12}$. En el ordenamiento de la R.I. de Irán, el nezām, para referirse al vali-ye faqih (el jurisperito islámico encargado del gobierno) se utilizan más bien los términos rahbar (guía/líder), rahbar-e enqelāb (guía/líder de la revolución), maqām-e rahbari (autoridad del guía/líder) o maqām-e mo ‘azzam-e rahbari (gran autoridad del guía/líder).

La Constitución empieza con un Preámbulo, en el que se afirma que:

«El proyecto del gobierno islámico (hukumat-e eslāmi) está fundado en la velāyat-e faqih, como presentada por el imam Jomeiní en el ápice de la opresión y represión del régimen despótico» ${ }^{13}$.

Y más adelante:

«A base de los principios de la velāyat-e amr y del imamato ininterrumpido (emāmat-e mostamarr), la Constitución prepara el terreno para el liderazgo (rahbari) del jurisperito islámico (faqih) dotado de todos los requisitos necesarios ( $\hat{y} \bar{a} m e$ ' ol-šarāyet) y que sea reconocido por el pueblo (mardom) como guía (rahbar) a fin de que sea garante del respeto por parte de las diferentes instituciones de sus deberes islámicos fundamentales».

Esta declara que la soberanía pertenece a Dios (art. 2 y 56). El Duodécimo imam, al-Mahdi, en ocultación desde el siglo IX, es el único legítimo representante de Dios sobre la tierra y, como afirmado por el art. 5, un artículo fundamental para nuestro análisis:

«Durante la ocultación de su excelencia el Duodécimo imam (Vali-e 'asr), en la R.I. de Irán la tutela (velāyat) y el imamato de la comunidad de los creyentes (ommat) está a cargo de un jurisperito islámico (faqih) justo, pío, consciente de las necesidades del tiempo, valiente, prudente y dotado de capacidades administrativas, que asume su tarea a base del art. 107».

Mientras que el primer rahbar no fue elegido y está indicado directamente por nombre en la Constitución en la persona del marŷa' al-taqlid Jomeiní

este se traduce con 'sistema'. Sin embargo, mi experiencia de estudio de la R.I. de Irán indica que sería más apropiado utilizar los términos ‘ordenamiento' o 'Estado'. Este es el caso, por ejemplo, del valor del termino en el nombre de una de las instituciones más importante del país, el Maŷma'-e Tašjis-e Maslahat-e Nezām (Consejo de Discernimiento de la Razón de Estado). Debido a la dificultad de trasladar la complejidad del termino (o más bien de su utilización en la R.I. de Irán), en este ensayo en la mayoría de los casos he preferido no traducirlo y dejarlo en persa.

12 Entre los estudiosos que se han interesado en el tema ‘islam y constitucionalismo’ en Irán destaca la labor de Said Amir Arjomand. Entres sus trabajos véase Said Amir Arjomand, After Khomeini: Iran Under His Successors, New York, Oxford University Press, 2009, en particular pp. 26-55, e "Islam and Constitutionalism since the Nineteenth Century: The Significance and Peculiarities of Iran" [en Íd, ed.: Constitutional Politics in the Middle East: With Special Reference to Turkey, Iraq, Iran and Afghanistan, Oxford and Portland Oregon, Hart Publishing, 2008].

${ }_{13}$ Todas las traducciones de la Constitución de la R.I. de Irán son del Autor del ensayo. 
(Preámbulo y art. 107), la tarea de elegir a los siguientes guías recae sobre la Asamblea de los Expertos del Liderazgo (Maŷles-e Jobregān-e Rahbari), cuyos 86 miembros son electos directamente por el pueblo y por sufragio universal por un periodo de ocho años; sin embargo, tienen que ser todos jurisperitos islámicos capaces de efectuar el iŷtihād (muŷtahed/faqih). La Asamblea tiene la tarea de identificar, entre todos los muytahed/faqih que cumplan con los requisitos de los artículos 5 y 109 de la Constitución, el que se destaca por uno o más de los requisitos mencionados por el art. 109 y de nombrarlo 'guía' (rahbar) de la República. En el caso de que no sea posible identificar a un jurisperito islámico que tenga esta preeminencia, la Asamblea elige como rahbar a uno de sus miembros (art. 107).

Cabe mencionar que cuando, el día 4 de junio de 1989, a la muerte del gran ayatola Jomeiní, la Asamblea elijo al actual guía, Ali Jamenei, el mismo no gozaba todavía ni de la posición de marŷa 'al-taqlid ni del calificativo de muŷtahid/faqih - que sin embargo hoy muchos le reconocen-, y entonces no cumplía con un requisito fundamental requerido por la primera Constitución (y tampoco con la enmendada). En efecto, en el mismo año, y tras la expresa voluntad del moribundo gran ayatola Jomeiní, la Constitución fue enmendada, a través de un largo debate concluido el día 28 de julio del 1989 en el que se enmendaron y añadieron 48 artículos, en particular con respecto a la función del rahbar y a la doctrina de la wilāyat al-faqih. Antes de la enmienda constitucional, los artículos 5 y 107 mencionaban la necesidad de que el liderazgo (rahbari) y la marŷa 'iyat del vali-e faqih fuesen reconocidos y aceptados por la (gran) mayoría de la población. Esta condición fue eliminada, dejando su elección como prerrogativa exclusiva de la Asamblea de los Expertos del Liderazgo. Además, la misma Asamblea puede destituir al guía en el caso de que este se vuelva incapaz de cumplir con sus obligaciones o pierda uno de los requisitos previstos por los artículos 5 y 109 (como mencionado por el art. 111). A parte por estos casos, el mandato del rahbar dura toda la vida. Por otro lado, el art. 107 menciona que:

«El guía (rahbar) es igual a todos los otros miembros del país (kešvar) ante la ley».

Las enmiendas de 1989 a la Constitución fortalecieron los poderes del rahbar. En efecto, con la enmienda del art. 110, que detalla sus prerrogativas y deberes, por una parte el ejercicio de los tres poderes del Estado (legislativo, ejecutivo y judiciario) fue puesto bajo la supervisión de la 'tutela absoluta del jurisperito islámico’ (velāyat-e motlaqe-ye faqih) (art. 57) y, por otra parte, fue eliminada la posibilidad de que, en lugar de un solo guía, se pudiese establecer un Consejo del Liderazgo (Šurā-ye Rahbari), definiendo así el oficio del rahbar como oficio exclusivamente monocrático. 
Además, a los poderes del rahbar previstos por la Constitución de 1979, que prevén que este tenga las siguientes prerrogativas:

-nombramiento de los jurisperitos islámicos (foqahā) miembros del Consejo Guardián (Šurā-ye Negahbānn ${ }^{14}$ (art. 110);

-nombramiento del jefe de la Judicatura (art. 110);

-nombramiento del jefe de Estado Mayor de Defensa, de los Comandantes Generales de las tres fuerzas armadas y del jefe de las Fuerzas de Policía (art. 110);

-nombramiento del jefe de los Guardianes de la Revolución (Sepāh-e Pasdaran) (art. 110);

-jefatura del Comando Supremo de las Fuerzas Armadas (art. 110); -declaración de guerra y paz (art. 110);

-destitución del presidente de la República a resultas de sentencia de la Corte Suprema (Divān-e 'Āli-ye Kešvar) o a voto del Parlamento (Asamblea Consultiva Islámica, Maŷlés-e Šurā-ye Eslāmi), teniendo en cuenta los intereses del país (masāleh-e kešvar) (art. 110);

-concesión del derecho de gracia y de reducción de penas (art. 110);

-nombramiento de dos representantes en el Consejo Estratégico para las Relaciones Exteriores (Šurā-ye Rāhbordi-ye Ravābet-e Jāreŷi) (art. 176);

-supervisión de la actuación de los tres poderes del Estado (art. 57);

con las enmiendas de 1989 se añadieron las prerrogativas de:

-determinación de las políticas generales del nezām de la R.I. de Irán después de haber consultado el Consejo de Discernimiento de la Razón de Estado (Maŷma'-e Tašjis-e Maslahat-e Nezām) (art. 110);

-coordinación de los tres poderes del Estado y solución de sus contrastes (art. 110);

-resolución, a través de la mediación del Consejo de Discernimiento de la Razón de Estado (Maŷma'-e Tašjis-e Maslahat-e Nezām), de las dificultades del nezām que no puedan ser resueltas a través de medidas ordinarias (art. 110);

-supervisión de la buena ejecución de las políticas generales del nezām (art. 110);

-convocación de referéndum (art. 110);

-nombramiento y revoca del presidente de la Radio y Televisión de

${ }^{14}$ Compuesto por doce miembros, seis jurisperitos islámicos capaces de capaces de efectuar el îytihäd y seis constitucionalistas, el Consejo evalúa las islamicidad y constitucionalidad de las leyes aprobadas por el Parlamento, además de la elegibilidad de los candidatos a las elecciones presidenciales y legislativas.

Araucaria. Revista Iberoamericana de Filosofia, Politica, Humanidades y Relaciones Internacionales, año $21, \mathrm{n}^{\circ} 41$. Primer semestre de 2019. Pp. 495-513. ISSN 1575-6823 e-ISSN 2340-2199 doi: 10.12795/araucaria.2019.i41.23 
la R.I. de Irán (artículos 110 y 175);

-revoca de las figuras institucionales que antes podía solo nombrar (art. 110).

Las enmiendas a la Constitución llevadas a cabo en 1989 representaron sobretodo el pasaje de la doctrina de la 'tutela del jurisperito islámico' (velāyat-e faqih) a la doctrina de la 'tutela absoluta del jurisperito islámico' (velāyat-e motlaqe-ye faqih).

¿Qué significado tiene este cambio? Significa que el rahbar puede emitir decretos gubernativos (ahkām-e homukati, plural de hokm-e humukati) a base del interés (maslahat) del nezām, del país (kešvar) o del islam y que están por encima de los decretos/mandamientos (hokm) derivados de la sharia (tanto de los 'primarios', avvaliye, como de los 'secundarios', sānaviye $)^{15}$. Este es un tema de gran relevancia y del que se hablará en el siguiente apartado.

Aquí es importante mencionar que, sin embargo, la misma revisión constitucional debilitó la autoridad del guía; por lo menos en términos teológicoreligiosos. Según el texto de la Constitución enmendada, de entre los requisitos previstos por el art. 109 para la asunción del cargo de rahbar, este debe poseer:

«1. la competencia (salāhiyat) científica necesaria para emitir fetuas (opiniones jurídicas) en los diferentes sectores del derecho islámico (fiqh);

2. la justicia y devoción necesarias para el liderazgo de la comunidad islámica (ommat-e eslām);

3. clara visión política y social, prudencia, coraje, capacidades administrativas y fuerza suficiente para el liderazgo.

En caso de que más de una persona cumpla con estos requisitos, aquella dotada de una más fuerte perspicacia (pišbini) en derecho islámico (fiqh) y en política tendrá la preferencia».

A tal respecto, a diferencia de la anterior versión del artículo, para la elección del guía no se requiere que este sea un marŷa 'al-taqlid, o sea goce del más alto nivel de competencia doctrinal y jurídica reconocida por parte de los primus inter pares del establishment religioso chí (formado por los otros marāŷa' al-taqlid y algunas familias conocidas como 'familias de ciencia' ${ }^{16}$ ) y sea efectivamente seguido por un importante numero de creyentes que le pagan

${ }^{15}$ En el derecho musulmán, los decretos secundarios (ahkām-e sānaviye) son aquellos decretos que hacen (temporalmente) lícito un comportamiento que de otra forma sería vedado por los 'decretos primarios' (ahkām-e avvaliye); o al revés hacen ilícito un comportamiento lícito. Estos están basados en la regla de la 'necesidad' o 'emergencia' ( $q \bar{a}$ 'ede-ye zarurat), que permite suspender un mandamiento/ decreto por un tiempo limitado, justamente el de la emergencia. Véase Domenico Parrello, Diritti delle minoranze e libertà religiosa: Il ruolo del fiqh nell'ordinamento giuridico iraniano, Roma, La Sapienza Orientale-Ricerche, 2008, pp. 50-51.

${ }^{16}$ En la historia moderna y, sobretodo, contemporánea del chiismo, estas familias han sido en gran medida representadas por alíes, descendientes de la Familia del Profeta. Véase Raffaele Mauriello, Descendants of the Familiy of the Prophet in Contemporary History: A Case Study, the Š̉ 'i Religious Establishment of al-Nağaf (Iraq), Roma-Pisa, Fabrizio Serra Editore-Rivista degli Studi Orientali, 2011. 
el sahm al-imām o jums, como mencionado más arriba. Sino que es suficiente ser muŷtahidlfaqih.

En su análisis sobre la génesis de la Constitución de la R.I. de Irán, GhamariTabrizi afirma que, en 1988 y en el curso de la revisión constitucional, Jomeiní llegó a la conclusión de que la condición de marŷa iyyat no era políticamente viable $\mathrm{y}$ hasta cita las palabras del mismo gran ayatola, en las que este recuerda como:

«Creí e insistí desde el principio en que la condición de marŷa'iyat no es necesaria. Un honrado moŷtahed que haya obtenido el refrendo de los respetados expertos [jobregān] debe de estar calificado, si el pueblo ha dado a los jobregān la autoridad para elegir a su líder [...] Esta era mi posición en la constitución originaria, pero mis compañeros insistieron en la condición de marŷa'iyat y yo accedí» ${ }^{17}$.

Además, cabe mencionar que los poderes del guía están condicionados al respeto de los intereses del nezām y de islam y que, siempre a partir de la enmienda constitucional del 1989, la actuación del rahbar está, por lo menos en principio y según la Constitución, puesta bajo la supervisión de la Asamblea de los Expertos del Liderazgo (Maŷles-e Jobregān-e Rahbari), cuyos miembros recordamos son todos jurisperitos islámicos capaces de efectuar el îytihād (muŷtahed/faqih) electos directamente por el pueblo por sufragio universal.

\section{La maslahat-e nezām}

A parte por la wilāyat al-faqih, el otro elemento que define y caracteriza la R.I. de Irán, su Constitución y la doctrina político-religiosa del país es la maslahat-e nezāmm ${ }^{18}$. Esta expresión se puede traducir con 'interés del sistema' o 'razón de Estado'.

El término maslahat (en persa, maslaha en árabe) deriva de una raíz que ocurre con frecuencia en el Alcorán, con significados como 'bueno', 'virtuoso', 'apropiado' y 'reconciliación' ${ }^{19}$. Sin embargo, el término maslahat como tal

${ }^{17}$ Behrooz Ghamari-Tabrizi, Islam and Dissent in Postrevolutionary Iran: Abdolkarim Soroush, Religious Politics and Democratic Reform, London, I.B. Tauris, 2008, pp. 48-49: «I believed and insisted from the beginning that the condition of marja iyat is not necessary. A righteous mojtahid who is endorsed by the respected experts [khobregan] ought to be qualified, if the people have given the khobregon the authority to choose their leader».

${ }^{18} \mathrm{Al}$ respecto, Mohsen Kadivar, Hukumat-e velā'i, Teherán, Našr-e Ney, 1998, afirma que al centro de la reelaboración de la velāyat-e faqih después de la Revolución por parte del mismo gran ayatola Jomeiní está precisamente su rearticulación de la noción de maslahat. La maslahat resulta fundamental también en la doctrina política e internacional del actual rahbar. Véase Seyed Mohammad Marandi, Raffaele Mauriello, "The Khamenei Doctrine: Iran's Leader on Diplomacy, Foreign Policy and International Relations" [en Nassef Manabilang Adiong, Deina Abdelkader, Raffaele Mauriello, eds.: Islam in International Relations: Politics and Paradigms, London \& New York, Routledge, 2019].

${ }^{19}$ Elsaid M. Badawi, Muhammad Abdel Haleem, Arabic-English Dictionary of Quranic Usage, 
nunca aparece en el Alcorán. En la actualidad política e institucional de la R.I. de Irán, el término se utiliza con el sentido de 'interés'/“conveniencia' y hasta 'razón [de Estado]'. Este principio jurídico parece ser controvertido en términos de derecho islámico chií. Según algunos estudiosos, si el concepto fue siempre aceptado por los musulmanes suníes, el mismo fue rechazado por los chiíes como bed 'at (innovación prohibida) ${ }^{20}$. Según otros estudiosos, al revés, la maslahat tiene un pasado muy largo en el derecho chií21 .

Para comprender la función esencial de la maslahat en la R.I. de Irán y en de la doctrina política de la wilāyat al-fqih, fundamental resulta una carta enviada por parte del primer rahbar, el gran ayatola Jomeiní, al entonces presidente de la República, Ali Jamenei, el día 7 de enero de 1988. En esta el fundador y teórico de la República islámica, en relación con la posibilidad de suspender los decretos derivados (ahkām-e far 'iye) de la sharia, afirmaba que:

«Tengo que precisar que el Gobierno (Hukumat), que es una branca ( $\check{o}$ ‘be) de la tutela absoluta (velāyat-e motlaqe) del Profeta de Dios, es uno de los decretos primarios (ahkām-e avvaliye) del islam y tiene prioridad sobre todos los decretos derivados (ahkām-e far'iye), incluido el rezo, el ayuno y el hâŷy (peregrinaje a la Meca). El gobernante (hākem) [islámico] puede demoler una mezquita o una casa que esté en el recurrido de una calle [en construcción] e indemnizar a su dueño. El gobernante (hākem) puede cerrar las puertas de una mezquita cuando sea necesario y puede demoler una mezquita [que esté causando un daño] en el caso de que no sea posible remover el daño [de otro modo]. El Gobierno (Hukumat) puede anular unilateralmente los contratos [sancionados] por la sharia que haya cerrado con la gente (mardom), en el caso de que estos contratos estén en contra de los intereses (masāleh) del país (kešvar) y del islam. Y puede impedir cualquier cuestión ( $a m r)$, tanto de culto ('ebādi) como de otro tipo (gheir-e 'ebādi), si su practica está en contra de los intereses (masāleh) del islam. El Gobierno (Hukumat) puede impedir temporáneamente el haŷy, que es una de las obligaciones [individuales] (farāyez, plural de farz) divinas [más] importantes, en el caso de que considere que [su practica] está en contra del interés (salāh) del país islámico (kešvar-e eslāmi)»22.

Leiden, Brill, 2008, p. 531.

${ }^{20}$ Por lo menos según Asghar Schirazi, The Constitution of Iran: Politics and the State in the Islamic Republic, translated by John O'Kane, London \& New York, I.B. Tauris, 1998, pp. 233 244. Sin embargo, Wael B. Hallaq, A History of Islamic Legal Theories, Cambridge, Cambridge University Press, 1997, pp. 112-113, e Íd, Shari 'a: Theory, Practice, Transformations, Cambridge, Cambridge University Press, 2009, pp. 504-508, afirma que la maslahat fue controvertida también entre los teóricos del derecho suníes pre modernos, antes de ser apropiada a finales del siglo XIX por Muhammad Rashid Rida, y a través de él por los reformistas islámicos, como pilar de su racionalización de las exigencias materiales de la modernidad. Finalmente, según Asma Afsaruddin, "Maslahah as a Political Concept" [en Mehrzad Boroujerdi, ed.: Mirror for the Muslim Prince: Islam and the Thoery of Statecraft, Syracuse NY, Syracuse University Press, 2013], pp. 16-44, como principio socio-político la maslahat siempre ha existido tanto entre los suníes como entre los chiíes.

${ }^{21}$ Véase Seyyed Sajad Izadhi, Maslahat dar feqh-e siyāsi-ye ši 'e, Teherán, Sazemān-e Enteshārāt-e Paŷuheshgāh Farhang va Andishe-ye Eslāmí, 1393/2014-15, en particular p. 18.

22 El texto integral en persa de la carta está disponible al siguiente enlace: http://www.imam- 
Estas palabras y el análisis desarrollado hasta ahora nos hacen llegar a la conclusión que, desde la perspectiva de la doctrina de la wilāyat al-faqih elaborada por el gran ayatola Jomeiní, e incorporada como eje fundamental de la Constitución de la R.I. de Irán, y con respecto a los elementos que definen y caracterizan la figura (y función) del rahbar, aun siendo una condicio sine qua non, hasta el calificativo de muŷtahid/faqih no es suficiente por si mismo para legitimar el poder del guía. Su función y poder están más bien justificados a base de su conocimiento y habilidad en responder a los desafíos y problemas con los que la República islámica se enfrenta en todos los ámbitos de la vida social, económica y política. Esto quiere decir que, como observado por Ghamari-Tabrizi ${ }^{23}$, la soberanía del vali-ye faqih no está legitimada (solo) por sus competencias en jurisprudencia islámica (feqh), sino más bien por su conocimiento y habilidad de responder a los problemas de su comunidad política y social. Esta conclusión parece ser confirmada por la prioridad que, como rahbar y teórico de la velāyat-e faqih, el gran ayatola Jomeiní dio a la maslahat (interés, o sea la política) sobre la feqāhat (derecho islámico, o sea a la religión) tanto en su gestión de la R. I. de Irán como en su misma teoría del gobierno islámico. En efecto, Jomeiní llevó su personal y original interpretación de la wilāyat al-faqih a sus extremas consecuencias a través de la primacía de la maslahat (la política) sobre la sharia (la religión). Él marcó este cambio en su doctrina añadiendo el término motlaqe (absoluto) y pasando de la doctrina de la velāyat-e faqih a la velāyat-e motlaqe-ye faqih ('tutela absoluta del jurisperito islámico').

Esta decisión, junto con la eliminación del requisito de marŷa iyat como hemos visto, el más alto nivel de competencia teológico-jurídica de la hierocracia chií unido al amplio reconocimiento por parte tanto de los primus inter pares como de los creyentes-, antes de la enmienda constitucional del 1989 necesario para el oficio de rahbar, ha llevado otra vez a la separación de la "tradicional" hierocracia religiosa representada por la marŷa 'iyat y el Estado $\mathrm{y}$, de ahí, a la preeminencia de lo político sobre lo religioso.

Además, y volviendo otra vez tanto a la Constitución, al mismo nezām, a los ahkām-e humukati (decretos gubernativos) y a la maslahat (interés, razón de Estado), por voluntad del gran ayatola Jomeiní, en 1989, tras las enmiendas a la Constitución, fue creado un especifico órgano encargado de resolver las crisis del Estado, el Maŷma'-e Tašjis-e Maslahat-e Nezām (Consejo de Discernimiento de la Razón de Estado). Como descrito por el art. 112 de la Constitución, este Consejo tiene la función institucional de mediar y resolver los contrastes jurídico-institucionales que surjan entre el Parlamento (la

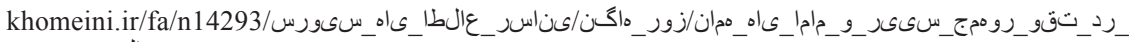
مقفف_تى النو_صوصخ

${ }_{23}$ Behrooz Ghamari-Tabrizi, Islam and Dissent in Postrevolutionary Iran: Abdolkarim Soroush, Religious Politics and Democratic Reform, London, I.B. Tauris, 2008, p. 145.

Araucaria. Revista Iberoamericana de Filosofia, Politica, Humanidades y Relaciones Internacionales, año $21, \mathrm{n}^{\circ} 41$. Primer semestre de 2019. Pp. 495-513. ISSN 1575-6823 e-ISSN 2340-2199 doi: 10.12795/araucaria.2019.i41.23 
Asamblea Consultiva Islámica, Maŷlés-e Šurā-ye Eslāmí) y el Consejo de Guardianes (Šurā-ye Negahbān) a través del discernimiento de la razón de Estado (maslahat-e Nezām) y, además, de servir de órgano consultivo al rahbar en la determinación de las políticas generales del nezām de la R.I. de Irán (art. 110). Al respecto, el art. 112 afirma que:

«El Consejo de Discernimiento de la Razón de Estado (Maŷma'-e Tašjis-e Maslahat-e Nezām) se reúne por orden del rahbar para determinar el interés (maslahat) [del nezām] en los casos en los que el Consejo de Guardianes ( ̌̌urāye Negahbān) considere que las disposiciones (mosavvabe) de la Asamblea Consultiva Islámica Maŷlés-e Šurā-ye Eslāmí) van en contra de los principios (mavāzin) de la sharia o de la Constitución y[, no obstante,] la Asamblea [Consultiva Islámica], teniendo en cuenta el interés (maslahat) del nezām, no confirme la opinión del Consejo de Guardianes (Šurā-ye Negahbān); y sirve de [órgano] de consultación en los asuntos en los que el rahbar les defiera y en las otras obligaciones previstas en esta Constitución. Los miembros permanentes y no permanentes del Consejo son nombrados por el maqām-e rahbari...».

O sea, la función del Consejo es de fortalecer la primacía de la maslahat (política) sobre la sharia (religión).

\section{La Oficina del Guía, o la geopolítica del rahbar}

Después de haber analizado el marco doctrinal y constitucional de la función del rahbar en la R.I. de Irán, y con el objeto de abordar el tema de la proyección de sus poderes en los diferentes órganos del Estado (nezām), en el territorio de la República y en el extranjero, es necesario tratar un aspecto esencial en el cumplimiento de las prerrogativas del guía en el ordenamiento de la República, o sea la zona gris (en términos constitucionales) constituida por sus representantes.

El rahbar ejerce sus funciones a través de una especifica oficina, la Oficina del Guía (Daftar-e Maqām-e Mo 'azzame-Rahbari). Aun no siendo expresamente prevista por la Constitución, esta menciona la posibilidad de que el rahbar pueda delegar parte de sus responsabilidades y poderes (ejtiy $\bar{a} r \bar{a} t)$ (art. 110). Sin embargo, el alcance de esta prerrogativa ha ido mucho más allá de lo previsible (constitucionalmente).

Este es el ámbito de los que se podrían definir 'los hombres del guía'. Y no es un caso si uno de los cuatro miembros permanentes de la oficina sea el ayatola Mohammad Golpaigani, ligado al actual rahbar a través de un vínculo matrimonial. Además, la Oficina cuenta con diez consejeros especiales ${ }^{24}$. Pero, y sobretodo, esta cuenta con un numero imprecisado de representantes del

\footnotetext{
${ }^{24}$ Véase Wilfried Buchta, Who Rules Iran? The Structure of Power in the Islamic Republic, Washington, The Washington Institute for Near East Policy-Konrad Adenauer Stiftung, 2000, pp. 46-47.
} 
guía (nemāyandehā-ye maqām-e mo 'azzam-e rahbari), que se estima ser de alrededor de 600 personas $^{25}$. Estos representantes se encuentran en ${ }^{26}$ :

- la prensa. Este es el caso sobretodo del periódico Keyhan (el más influyente entre los conservadores), cuyo director está nombrado directamente por el guía, y de Ettela'at (el periódico en activo más antiguo de la prensa iraní $)^{27}$;

- el poder ejecutivo. Todos los ministerios y muchos departamentos de la Administración Pública tienen un despacho reservado al correspondiente nemāyandehā-ye maqām-e mo 'azzam-e rahbari;

- el ejercito y los servicios de inteligencia: fuerzas armadas (ejercito, marina, aeronáutica y sus servicios de inteligencia), Pasdaranes (y sus servicios de inteligencia), fuerzas Basiŷi (desde 2007 bajo control directo de los Pasdaranes) y de policía;

- las universidades y las hausāt (plural de hausa, los centros de enseñanza del diseño curricular clásico de formación teológico-jurídica). Todas ellas tienen un despacho reservado para el correspondiente nemāyande-ye vali-ye faqih. Además, el guía nombra directamente al director de la hausa de Qom;

- el ámbito religioso. Este es el caso en particular del Consejo de los Imames para el Rezo del Viernes, que nombra a los 'olemā que dirigen el rezo del viernes de Teherán y de todas las ciudades del país. Estos 'olemā además tienen la función de representantes del rahbar en las respectivas provincias donde operan;

- las organizaciones religiosas. El guía nombra a los directores de la Organización para la Propaganda Islámica (Sāzemān-e Tablighāt-e Eslāmi), la Organización para el Peregrinaje (Sāzemān-e Haŷŷ va Ziyārat), la Sociedad para la Reconciliación entre las Escuelas Jurídicas Islámicas (Maŷma'-e ỹahāni-ye Barā-ye Taqrib-e Bein-e Mazāheb-e Eslāmi) y la Asamblea para le Gente de la Casa del Profeta (Maŷma'-e Jahāni-ye Barā-ye Ahl-e Beit);

- los centros culturales de la R.I. de Irán en el extranjero y las consejerías culturales de sus embajadas, que operan bajo la dirección de la Organización de Cultura y Relaciones Islámicas (Sāzemān-e Farhang va Ertebātāt-e Eslāmi), cuyo director es nombrado por el guía. En este caso, estamos en el ámbito de la propaganda y política estera de la Oficina del Guía (Daftar-e Maqām-e Mo 'azzame- Rahbari), esta última llevada a cabo también a través de comisiones y organismos ad hoc, como el Consejo Estratégico para las Relaciones Exteriores (Šurā-ye Rāhbordi-ye Ravābet-e Jāreŷy $)^{28}$;

${ }^{25}$ Ibíd., p. 47.

${ }^{26}$ Ibíd., p. 49.

27 Amir Taheri, "Iran's Oldest Daily Blows out 90 Candles", Asharq Al-Awsat, 24 de noviembre de 2015. Disponible al siguiente enlace: https://eng-archive.aawsat.com/amir-taheri/news-middle-east/ irans-oldest-daily-blows-out-90-candles.

${ }^{28}$ Establecido en 2006 para salir -a favor del rahbar- del callejón sin salida del enfrentamiento institucional (y constitucional) entre el guía (Jamenei) y el presidente (Ahmadineyad) ocurrido en el

Araucaria. Revista Iberoamericana de Filosofia, Política, Humanidades y Relaciones Internacionales, año $21, \mathrm{n}^{\circ} 41$. Primer semestre de 2019. Pp. 495-513. ISSN 1575-6823 e-ISSN 2340-2199 doi: 10.12795/araucaria.2019.i41.23 
- los directores, representantes y miembros de consejos de administración de las bonyād (fundaciones "religiosas") 29 , como: la Fundación del Santuario del Imam Reza (Āstān-e Qods-e Razaví) en Mashhad, la Fundación 15 de Jordad (Bonyād-e Pānzdah-e Jordād) en Qom, la Fundación los Desheredados y Discapacitados de la Revolución Islámica (Bonyād-e Mostaz'afān va ŷānbāzān-e Enqelāb-e Eslāmi) y la Fundación de los Mártires y de los Asuntos de los Abnegados (Bonyād-e Šahid va Omur-e Isārgarān).

Más allá de la Oficina del Guía, y en parte en superposición con esta, cabe mencionar que la proyección de los poderes del rahbar también se realiza en parte a través del Beit-e Rahbar, o sea la familia del Guía (sus hijos, yernos, nietos, etc.). En la marŷa 'iyat, la familia del marŷa' desempeña una función clave como "puerta" de acceso este ${ }^{30}$. Este fue el caso, por ejemplo, tanto de la familia del marŷa 'Abulqasem al-Ju'í, la más importante 'fuente de emulación' de los años 1970 y 1980 junto con Jomeiní, y también es el caso de la familia del marŷa 'Ali al-Sistani, el más importante actualmente a nivel internacional junto con Jamenei. Sin embargo, cabe mencionar que el Beit-e Jamanei no es el tradicional beit de los marāŷa', como era el caso del mismo primer guía de la República, el gran ayatola Jomeiní. Jamenei parece haber conscientemente tenido su familia directa (hijos, nietos, etc.) lejos de las funciones de poder tanto de su marŷa 'iyat como de su cargo de rahbar ${ }^{31}$. Al respecto, en cierta medida se puede afirmar que Jamenei haya, voluntariamente o menos, operado una de-carismatización del beit.

Consejo Supremo para la Seguridad Nacional (Šurā-ye 'Āli-ye Amniat-e Melli).

${ }^{29}$ Un caso ejemplar es el del Āstāne Qods-e Razavi, en la ciudad de Mashhad. Hoy en día capital de una de las tres provincias en las que ha sido recientemente dividida administrativamente la región histórica del Jorasán, con doce millones de visitantes al año Mashhad es uno de los más grandes centros de peregrinaje al mundo. La ciudad acoge la tumba y el mausoleo del imam Reza, el único imam sepultado en Irán. El Āstāne Qods-e Razavi es la fundación (waqf) que administra el mausoleo. La Fundación está considerada como la tercera organización económica del país, con participaciones en los principales sectores de la economía iraní, y posee: el $60 \%$ de los terrenos de la ciudad, más de 400000 hectáreas de tierras agrícolas, varias fábricas, hospitales, hoteles, editoriales, etc. Hablamos de una fundación cuyo valor estimado supera los 20000 millones de dólares y con una facturación anual estimada en más de 130 millones de dólares. El cargo de administrador de la fundación (motevalí) fue ejercido desde la revolución (1979) hasta su fallecimiento en el 2016 por el ayatola Abas Vaez-Tabasí, nombrado por Jomeiní y confirmado por su sucesor, Jamenei. Pues bien, la hija del ayatola VaezTabasí está casada con Hasan, hijo de Jamenei. Este vínculo matrimonial entre las dos familias fue además fortalecido por el matrimonio entre Naser, hijo de Vaez-Tabasí, y una de las hijas de Jamenei. Véase Bernard Hourcade, Géopolotique de l'Iran, Paris, Armand Colin, 2010, p. 53.

${ }^{30}$ Véase Raffaele Mauriello, Descendants of the Familiy of the Prophet in Contemporary History: A Case Study, the Šı ‘ $\imath$ Religious Establishment of al-Nağaf (Iraq), Roma-Pisa, Fabrizio Serra EditoreRivista degli Studi Orientali, 2011.

${ }^{31}$ Sin embargo, este no es el caso de sus yernos, que, como hemos visto, han desempeñado funciones importantes tanto en su Oficina como en la dirección de algunas fundaciones religiosas. 


\section{Los otros foqahā del nezām}

A parte el caso del rahbar, cabe mencionar que la institucionalización de la doctrina de la wilāyat al-faqih ha creado otros "jurisperitos islámicos de Estado". En efecto, la Constitución prevé que algunos miembros del nezām sean obligatoriamente muŷtahid/faqih:

- todos los 86 miembros de la Asamblea de los Expertos del Liderazgo (Mầles-e Jobregān-e Rahbari):

- los seis jurisperitos islámicos miembros del Consejo Guardián (̌̌urā-ye Negahbān) (art. 91);

- el jefe de la Judicatura (art. 157);

- el presidente de la Corte Suprema (Divān-e 'Āli) y el Fiscal General (Dādsetān-e Koll) (art. 162).

Además, el ordenamiento prevé que el ministro de Inteligencia ${ }^{32}$ sea un muŷtahidlfaqih y la "Ley sobre los requisitos para la selección de los jueces" prevé que, de entre los requisitos requeridos para ejercer la función de juez, los candidatos sean muŷtahid/faqih; o que, de lo contrario, reciban el permiso para ejercer la función por parte del jefe de la Judicatura (antes de la enmienda constitucional de 1989 el permiso lo emitía el Supremo Consejo de Justicia) $)^{33}$.

Un último caso de relieve respecto a la institucionalización del oficio de los 'olem $\bar{a}$ (jurisperitos islámicos, no necesariamente capaces de efectuar el $i \hat{y} t i h \bar{a} d$ ) en la R.I. de Irán que merece ser mencionado es el del Tribunal Especial para los Jurisperitos Islámicos (Dādgāh-e Viŷe-ye Ruhāniyat) ${ }^{34}$. Este tribunal, establecido en 1987 por decreto directo del primer rahbar, el gran ayatola Jomeiní, y que muchos estudiosos consideran inconstitucional, tiene la función de investigar y sancionar los crímenes -también "ideológicos"cometidos por los 'olemāa. Aquí su mención es importante porque representa una más de las instancias de institucionalización del oficio de los 'olemā.

\footnotetext{
${ }^{32}$ Iran's Ministry of Intelligence and Security: A Profile, Washington, Library of Congress, 2012, p. 1. Disponible al siguiente enlace: https://fas.org/irp/world/iran/mois-loc.pdf.

33 Domenico Parrello, Diritti delle minoranze e libertà religiosa: Il ruolo del fiqh nell'ordinamento giuridico iraniano, Roma, La Sapienza Orientale-Ricerche, 2008, pp. 39-42. En la realidad, la mayoría de los jueces en la R.I. de Irán no son muŷtahid/faqih a causa de la falta de candidatos que posean este "título".

34 Véase Mirjam Künkler, "The Special Court of the Clergy (Dādgāh-Ye Vizheh-Ye Ruhāniyat) and the Repression of Dissent Clergy in Iran" (May 13, 2009) [disponible en SSRN: https://ssrn.com/ abstract=1505542. Publicado en Said Arjomand and Nathan Brown (eds.), Constitutionalism, the Rule of Law and the Politics of Administration in Egypt and Iran, Albany, SUNY Press, 2012], pp. 57-100.
} 


\section{Conclusiones}

La Constitución de la R. I. de Irán no describe el rahbar como la máxima autoridad religiosa del país, sino como la máxima autoridad política. En tal sentido, desde una perspectiva estrictamente jurídica, el poder del guía es en gran parte comparable al poder de los jefes de Estado de muchos países democráticos ${ }^{35}$. La diferencia fundamental está en la dimensión también "religiosa" de su oficio y, sobretodo, en su capacidad de proyección geopolítica de sus poderes a través de la Oficina del Guía (Daftar-e Maqām-e Mo 'azzameRahbari); un elemento controvertido, no obstante, en términos estrictamente constitucionales. Su legitimidad está basada en su función de árbitro y garante de los intereses del Estado (nezām), más que en la dimensión "religiosa" de su oficio.

El análisis desarrollado en el presente ensayo indica que, por muchos versos, con la Revolución (1978-79) y el nacimiento de la R.I. de Irán, y sobretodo a través de la enmienda constitucional de 1989, se ha puesto en marcha un proceso de secularización de la doctrina de la wilāyat al-faqih, cuyo carácter político ha ido tomando la delantera ante el aspecto religioso.

El análisis de la institucionalización de la wilàyat al-faqih y de las prerrogativas y funciones del rahbar en la R.I. de Irán parecen indicar que, en la República islámica legitimidad religiosa y legitimidad popular (o sea política) legitiman una a la otra. En efecto, esta doble vertiente de la legitimidad del nezām se fundamenta y trae origen de la doctrina política del mismo gran ayatola Jomeiní, que, a pesar de que insistió en el fundamento islámico de la constitución, también consideraba el voto del pueblo esencial para la legitimidad del gobierno islámico ${ }^{36}$.

Parece interesante que tanto los autores de la Constitución de 1979 como de las enmiendas de 1989 hayan decidido evitar la utilización de la expresión vali-ye faqih para referirse al Jefe de Estado de la República islámica de Irán, optando más bien por el termino rahbar, guía/leader.

El ordenamiento (nezām) instaurado por la Revolución islámica ha

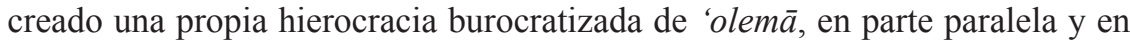
contraste con la tradicional hierocracia informal constituida por el sistema de la marŷa 'iya (representado actualmente por personajes como los gran ayatolas

${ }^{35}$ Y no es una casualidad si muchos apuntan a un origen francés tanto del marco de las amplias prerrogativas del Jefe de Estado de la R.I. de Irán como del Consejo de Guardianes -o sea la constitución de la Quinta República, con sus extraordinarios poderes ejecutivos concedidos al presidente y de revisión judicial concedidos al Consejo Constitucional-; como es el caso de Behrooz Ghamari-Tabrizi, Islam and Dissent in Postrevolutionary Iran: Abdolkarim Soroush, Religious Politics and Democratic Reform, London, I.B. Tauris, 2008, pp. 41-49.

${ }^{36}$ Al respecto, véase el análisis desarrollado por Behrooz Ghamari-Tabrizi, Islam and Dissent in Postrevolutionary Iran: Abdolkarim Soroush, Religious Politics and Democratic Reform, London, I.B. Tauris, 2008, p. 47. 
Ali al-Sistani y Mohammad Said al-Hakim en al-Nayaf o Fazel Lankarani y Safi Golpaigani en Qom). También un marcado proceso de institucionalización y burocratización del oficio de rahbar. 


\section{Referencias bibliográficas:}

Asma Afsaruddin, "Maslahah as a Political Concept" [en Mehrzad Boroujerdi, ed.: Mirror for the Muslim Prince: Islam and the Thoery of Statecraft, Syracuse NY, Syracuse University Press, 2013].

Said Amir Arjomand, After Khomeini: Iran Under His Successors, New York, Oxford University Press, 2009.

Said Amir Arjomand, "Islam and Constitutionalism since the Nineteenth Century: The Significance and Peculiarities of Iran" [en Íd, ed.: Constitutional Politics in the Middle East: With Special Reference to Turkey, Iraq, Iran and Afghanistan, Oxford and Portland Oregon, Hart Publishing, 2008].

Said Amir Arjomand, The Turban for the Crown: The Islamic Revolution in Iran, New York, Oxford University Press, 1988.

Alessandro Bausani, L'Islam, Milano, Garzanti, 1999.

Yahya Christian Bonaud, Uno gnóstico sconosciuto del XX secolo. Formazione e opere dell'Imam Khomeinî, a cura di Eugenio Tabano, Trieste, il Cerchio, 2010 [trad. de: L'Imam Khomeyni, un gnostique méconnu du XXe siècle. Métaphysique et théologie dans les ouvres philosophiques et spirituelles de l'Imam Khomeyni, Ed. Al-Bouraq, Beirut, 1997].

Wilfried Buchta, Who Rules Iran? The Structure of Power in the Islamic Republic, Washington, The Washington Institute for Near East PolicyKonrad Adenauer Stiftung, 2000.

Elsaid M. Badawi, Muhammad Abdel Haleem, Arabic-English Dictionary of Quranic Usage, Leiden, Brill, 2008.

Bernard Hourcade, Géopolotique de l'Iran, Paris, Armand Colin, 2010.

Behrooz Ghamari-Tabrizi, Islam and Dissent in Postrevolutionary Iran: Abdolkarim Soroush, Religious Politics and Democratic Reform, London, I.B. Tauris, 2008.

Wael B. Hallaq, Shari'a: Theory, Practice, Transformations, Cambridge, Cambridge University Press, 2009.

Wael B. Hallaq, A History of Islamic Legal Theories, Cambridge, Cambridge University Press, 1997.

Mohsen Kadivar, Hukumat-e velā'i, Teherán, Našr-e Ney, 1998.

Seyyed Sajad Izadhi, Maslahat dar feqh-e siyāsi-ye ši ‘e, Teherán, Sazemān-e Enteshārāt-e Paŷuheshgāh Farhang va Andishe-ye Eslāmí, 1393/2014-15. Mirjam Künkler, "The Special Court of the Clergy (Dādgāh-Ye Vizheh-Ye Ruhāniyat) and the Repression of Dissent Clergy in Iran" (May 13, 2009) [disponible en SSRN: https://ssrn.com/abstract=1505542. Publicado en Said Arjomand and Nathan Brown, eds.: Constitutionalism, the Rule of Law and the Politics of Administration in Egypt and Iran, Albany, SUNY Press, 2012, pp. 57-100]. 
Seyed Mohammad Marandi, Raffaele Mauriello, "The Khamenei Doctrine: Iran's Leader on Diplomacy, Foreign Policy and International Relations" [en Nassef Manabilang Adiong, Deina Abdelkader, Raffaele Mauriello, eds.: Islam in International Relations: Politics and Paradigms, London \& New York, Routledge, 2019].

Raffaele Mauriello, Descendants of the Familiy of the Prophet in Contemporary

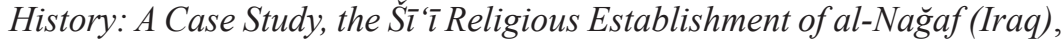
Roma-Pisa, Fabrizio Serra Editore-Rivista degli Studi Orientali, 2011.

Hamid Mavani, “Analysis of Khomeini's Proofs for al-Wilaya al-Mutalqa [sic] (Comprehensive Authority) of the Jurist" [en Linda S. Walbridge, ed.: The Most Learned of the Shi 'a: The Institution of the Marja 'al-Taqlid, New York, Oxford University Press, 2001].

Domenico Parrello, Diritti delle minoranze e libertà religiosa: Il ruolo del fiqh nell'ordinamento giuridico iraniano, Roma, La Sapienza OrientaleRicerche, 2008.

Abdulaziz Abdulhussein Sachedina, The Just Rules in Shi'ite Islam: The Comprehensive Authority of the Jurut in Imamite Jurisprudence, New York, Oxford University Press, 1988.

Asghar Schirazi, The Constitution of Iran: Politics and the State in the Islamic Republic, translated by John O'Kane, London \& New York, I.B. Tauris, 1998.

Amir Taheri, "Iran's Oldest Daily Blows out 90 Candles", Asharq Al-Awsat, 24 de noviembre de 2015.

Linda S. Walbridge (ed.), The Most Learned of the Shi 'a: The Institution of the Marja ' al-Taqlid, New York, Oxford University Press, 2001. 
